\title{
Enhancing Longevity of Pacemakers Through Reprogramming. Underutilization and Cost-Effectiveness
}

\author{
A ntonio Luiz Pinho Ribeiro, Leonor Garcia Rincón, Bruna Guimarães O liveira, Cleonice Carvalho Mota, \\ Marco Túlio Bacarini Pires \\ Belo Horizonte, MG - Brazil
}

\begin{abstract}
Objective -This study was performed to observe the number of pacemakers that had never been reprogrammed after implantation, and the effect of optimised output programming on estimated longevity of pulse generators in patients with pacemaker
\end{abstract}

Methods - Sixty patients with Teletronics Reflex pacemakers were evaluated in a pacemaker clinic, from the time of the beginning of its activities, in June 1998, until March 1999. Telemetry was performed during the first clinic visit, and we observed how many pulse generators retained nominal output settings of the manufactures indicating the absence of reprogramming until that date. After evaluation of the capture threshold, reprogramming of pacemakers was performed with a safety margin of 2 to 2.5:1, and we compared the estimated longevity based on battery current at the manufacturer's settings with that based on settings achieved after reprogramming.

Results - In 95\% of the cases, the original programmed setting was never reprogrammed before the patients attended the pacemaker clinic. Reprogramming the pacemaker prolonged estimated pulse generator life by $19.7 \pm 15.6$ months $(35.5 \%)$.

Conclusion - The majority of the pacemakers evaluated had never been reprogrammed. Estimated pulse generator longevity can be prolonged significantly, using this simple, safe, efficacious, and cost-effective procedure.

Key words: artificial cardiac stimulation, telemetry, costeffectiveness

Hospital das Clínicas da Faculdade de Medicina - UFMG

Mailing address: Antonio Luiz Pinho Ribeiro - Rua Campanha, 98/101 - 30310-770

- Belo Horizonte, MG - Brazil - E-mail: antonior@ net.em.com.br
Electric pacing of the heart began around forty years ago and stands out as one of the major achievements in cardiac therapy in the 20th century, combining both physiologic knowledge and technologic advances in the treatment of potentially disabling and fatal bradyarrhythmias. Currently, about 400 thousands pacemakers are implanted every year world-wide ${ }^{1}$, and $50 \%$ of pacemaker recipients are still alive when the pulse generator lifespan is over, making them eligible for a new pulse generator. Therefore, the prolongation of the life of pulse generators has been considered desirable and cost-effective because it would postpone a second surgical intervention, eliminating the expense of new generators units ${ }^{1,2}$.

Pacemaker longevity is defined as the interval between implantation and detection of the end of pulse generator life, which is determined by the energy consumption of the pulse generator and the deliverable capacity of its power source ${ }^{3,4}$. The deliverable capacity of the power source is fixed for a certain battery, but energy consumption depends on both fixed parameters and physiologic and programmable variables. Therefore, the determinants of the consuption are: programmable output parameters of the system (voltage and pulse width), the frequency and percentage of pacing, the lead impedance, and the static energy drain of the system. Although programming the pacing rate and $\mathrm{AV}$ interval can optimise the battery energy drain, the most important parameters that are available to optimise the longevity of a pacing system are stimulation voltage and pulse width ${ }^{2}$.

Original settings programmed by the manufacturer are chosen to provide for a sufficient safety margin during the subacute period when stimulation thresholds typically rise. However, most leads used today enable stimulation with reduced output parameters of pacing, resulting in significant reduction in energy drain and, therefore, prolongation of pulse generator life. Thus, reprogramming a pacemaker after a subacute period, according to an obtained stimulation threshold, was a desirable practice recommended in most basic textbooks and by international guidelines ${ }^{1,3-6}$. However, the majority of pacemakers remain at the original programmed 
settings and are never reprogrammed during their entire lifespan ${ }^{7,8}$. This study was performed to observe the number of pacemakers that had never been reprogrammed after implantation and the effect of optimised output programming on the estimated longevity of pulse generators.

\section{Methods}

Sixty patients with Teletronics Reflex pacemakers were evaluated in the Pacemaker Evaluation and Control Laboratory, of Hospital das Clínicas da UFMG, Belo Horizonte, Brazil, from the beginning of its activities, in June 1998 until March 1999. We chose patients who received a pacemaker of this make and model because the software of the programmer provides, in this case, an automatic estimate of generator longevity. The original algorithms used to calculate estimated longevity considers battery voltage, pulse width, frequency and stimulation mode, and cell and lead impedance. Patients who received pacemakers of other makes and models were excluded because no method was available to estimate longevity of the generator using the same algorithms used by the Reflex model.

Most patients (75\%) underwent pacemaker implantation in other clinics, coming to HCUFMG for regular followup control. Other patients underwent pacemaker implantation at in HCUFMG, before laboratory activities began. Fifty three patients received VVI pacemakers 8218 and 8220 , whereas only seven received DDD pacemaker 8224 . Estimated longevity of VVI pacemakers 8218 and 8220 is six years and that of the DDD 8224 generator is five years. One patient with a DDD 8224 pacemaker was receiving, by unknowing causes, single-chamber pacemaker stimuli. Only $3(5 \%)$ had epicardial pacemakers; it was the first pacemaker implantation for $32(56.1 \%)$ patients. All the 60 patients $(58 \pm 19$ years, 18 men) were included in systematic follow-up, according to a protocol based on international recommendations ${ }^{6}$. Telemetry was performed using Teletronics programmer model 9602. After measuring the threshold command, reprogramming of pulse amplitude and width was performed, with a safety margin of 2 to 2.5:1 (according to Barold et al.' recommendation ${ }^{9}$ ). Safety margin was defined according to the pulse amplitude voltage, with programming values of voltage $100 \%$ to $150 \%$ above the threshold obtained for a certain pulse width. All patients were still in clinical follow-up in March 1999, and no complications were reported related to output settings reprogramming.

Statistical analysis was carried out using Minitab software version 11. Data were expressed average \pm standard deviation. Analysis for differences in the groups' average was performed using Student's t test for paired and unpaired data, according to the situation.

\section{Results}

Values of parameters reached before and after programming are shown on Table I. Stimulation threshold with a pulse width of $0.375 \mathrm{~ms}$ was $0.7 \pm 0.3$ volts. Output settings were found in 57 patients (95\%), indicating the absence of repro- gramming in most patients. Patients had their pacemaker for about $36.0 \pm 25.9$ months when they first came to the laboratory, and their additional longevity was estimated at 52.7 \pm 21.8 months. Optimal programming enhanced predicted longevity to $72.0 \pm 32.7$ months $(\mathrm{p}<0.001)$, prolonging the generator lifespan(fig. 1) by $19.7 \pm 15.6$ months (35.5\%). Benefit was greater when reprogramming was done within 24 months after implantation $(25.8 \pm 16.0$ versus $13.2 \pm 12.8$ months, $p=0.001$ ).

\section{Discussion}

Enhancing the longevity of pulse generators units has been, since the beginning, an important aim of artificial cardiac stimulation ${ }^{10}$. Chemical energy available in the battery is consumed by its regular use and is depleted after a variable period, but all the other devices of the system of cardiac stimulation are designed to last indefinitely ${ }^{3}$. When the voltage output falls to a level in which cardiac stimulation can no longer be maintained, the battery needs to be replaced and, therefore, the entire pulse generator. Major technological advances have been made in the last decades that have improved the development of batteries and the efficiency of pulse generators, enabling progressive miniaturisation of pacemakers and enhancing generator lifespan. However, advances in lead design have led to the most dramatic improvements in pacing system longevity, as they enable cardiac stimulation at low energy ${ }^{11-14}$.

Benefits obtained from modern leads as the result of decreased stimulation thresholds are dependent on repro-

\begin{tabular}{|c|c|c|c|}
\hline \multicolumn{4}{|c|}{$\begin{array}{l}\text { Table I - Cardiac stimulation parameters obtained through } \\
\text { telemetry in } 60 \text { patients with Teletronics Reflex pacemakers before } \\
\text { and after power output reprogramming. }\end{array}$} \\
\hline & Before & After & $\mathrm{p}$ \\
\hline Pulse amplitude (vollts) & $5.0 \pm 0.7$ & $3.2 \pm 1.2$ & 0.000 \\
\hline Pulse width (milliseconds) & $0.395 \pm 0.130$ & $0.319 \pm 0.127$ & 0.000 \\
\hline Estimated longevity (months) & $52.7 \pm 21.8$ & $72.0 \pm 32.7$ & 0.000 \\
\hline
\end{tabular}

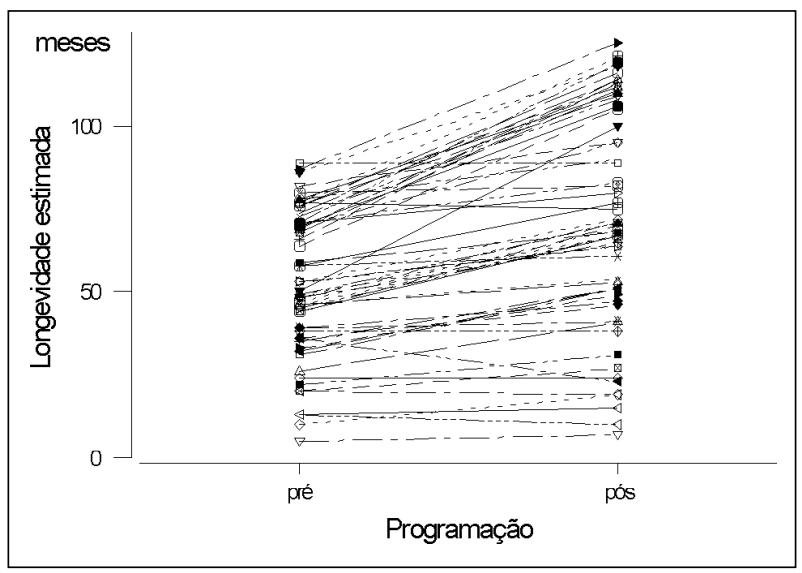

Fig. 1 - Estimated pulse generator unit longevity before and after reprogramming. 
gramming of the pulse generator to a lower stimulation voltage: original settings programmed by the manufacturer are quite generous in an effort to provide an adequate safety margin, even during the subacute period, which is characterised by a transitory and significant increase in the stimulation threshold. However, chronic thresholds are characteristically stable, with circadian oscillation lower than $50 \%{ }^{4}$. Prospective studies published early in the 90s proved that, through programming of output settings, generator unit lifespan could be significantly enhanced ${ }^{15}$ safely and without loss of command as verified by telemetry and 24-hour Holter monitoring ${ }^{14}$.

However, it is known that pacemakers are frequently never reprogrammed after implantation: in the United States, Griffin et al. ${ }^{7}$ acknowledge that only $43 \%$ of patients with pacemakers were followed-up regularly in a pacemaker clinic in 1986, whereas in 1991, in Germany, Irnich et al. ${ }^{8}$ revealed that $52.1 \%$ of the multiprogrammable generators taken postmortem were still programmed at the manufacturer's settings. We do not have estimates in our country, but we verified that almost all our patients did not have their pacemaker reprogrammed before evaluation in the pacemaker laboratory, even though the mean time of implantation was $36.0 \pm 25.9$ months. These data are in accordance with the data based on methods used in several clinics where pacemakers are followed up through simple evaluations, using only the miniclinic, X-ray, and electrocardiogram. This practice deprives patients with pacemakers from using resources for reprogramming pulse generators, with a potential impact on the patient's quality of life and, as previously mentioned, on generator unit longevity.

We verified a significant increase in generator longevity through reprogramming, in that, the effect is greater when it is performed within 24 months of implantation. However, even in patients where pacemakers had been implanted for more than two years, output settings reprogramming may result in significant increase in estimated longevity. The medical and economical impact of this strategy cannot be ignored. Crossley et al. ${ }^{2}$, while studying the efficiency and cost-effectiveness of reprogramming in 122 patients in 1996 reported a potential extension in the time of generator replacement of $4.25 \pm 2.14$ years, when intervention was performed within three to six months after implantation. Taking into consideration the charges for medical visits in the United States health system, the authors calculated that the mean cost of this benefit was $\$ 110$ per patient, which is a highly cost-effective ${ }^{2}$. Gillis et al. ${ }^{16}$ estimated that, only by reprogramming pulse width to $3.5 \mathrm{~V}$ (as opposed to the $5.0 \mathrm{~V}$ of the manufacturer), the estimated savings obtained per patient is between $\$ 2139$ and $\$ 4584$, depending on the type of pacemaker used. For these authors, total deliverable battery capacity is important ${ }^{16}$. They have estimated that the savings to the health system from using pacemakers with longer lifespans versus those with shorter lifespans is $\$ 424$ millions, considering the different pulse generators and the variable stimulation amplitudes.

Economical advantages are only a part of the potentially attainable benefits of systematically following up pa- tients with pacemakers. Although significant dysfunctions in the stimulation system do not occur frequently, they may be responsible for sudden pacemaker failure, and occasionally for a patient's death ${ }^{17}$. Griffin et al. ${ }^{7}$, while studying 1065 patients followed up for a year, found 61 systems $(5.7 \%)$ with significant dysfunction, of which, $85 \%$ required pacemaker reprogramming. Furthermore, in our country, patients with pacemakers feel stigmatised, restricting themselves harmfully and unnecessarily. They skip regular follow up, where they can find a multidisciplinary team, can settle their doubts, and can make use of all the benefits cardiac stimulation can provide.

Although the conclusions of this study are sustained by previous studies in the literature, some limitations must be acknowledged. The studied cohort was formed by patients who sought or were referred for follow-up to the Hospital das Clínicas of UFMG, rather than by patients, randomly chosen, who received pacemakers in Minas Gerais state. Patients who were regularly followed up elsewhere were not included in this study; therefore, we cannot affirm that systematic reprogramming has not been performed in other groups of patients. However, it is irrefutable in this study that the high number of patients observed whose pacemakers had never been reprogrammed indicates that reprogramming is not a common practice in our country. Different formulas exist for calculating estimated generator longevity, and in all of them some variables are arbitrarily defined ${ }^{9}$. Thus, although the relationship between total battery capacity and its consumption is known, no real guarantees exist that the real longevity will be the same as the estimated, also because changes in artificial stimulation conditions may occur during follow-up. In the present study, the manufacturer's algorithm was used to calculate the estimated longevity, which was unfortunately not available for the authors of this study. Finally, the Reflex model is no longer available on the market, and new pulse generators with several new programming resources are used routinely.

Among them, the systems with AutoCapture ${ }^{\mathrm{TM}}$ function stand out. They allow for the output to be automatically adjusted based on automatic evaluations of pacing threshold, making the most of optimised output ${ }^{18}$. When AutoCapture ${ }^{\mathrm{TM}}$ function (or a similar mechanism) be available in all models, manual output reprogramming will no longer be necessary. However, presently, few pacemaker models have this resource, and a great number of pulse generators are implanted that do not have the AutoCapture ${ }^{\mathrm{TM}}$ function. Thus, pulse generator output setting programming, with the purpose of enhancing lifespan, is formally indicated as part of postimplantation follow-up.

In conclusion, we observed that almost all Reflex pulse generators had never been reprogrammed in patients who were sent to a new pacemaker clinic, indicating that follow-up was not properly conducted. Systematic reprogramming of output settings significantly enhanced pulse generator lifespan. This simple and valuable procedure with a favourable cost-benefit relationship must be performed routinely during follow-up after pacemaker implantation in which AutoCapture ${ }^{\mathrm{TM}}$ is not available. 


\section{References}

1. Barold SS,Zipes DG. Cardiac pacemakers and antiarrhythmic devices. In: Braunwald E., editor. Heart Disease. A Textbook of Cardiovascular Medicine. $2^{\text {nd }}$ ed Philadelphia: WB Saunders, 1997: 705-41.

2. Crossley GH, Gayle DG, Simmons TW, et al. Reprogramming pacemakers enhances longevity and is cost effective. Circulation 1996; 94(suppl. II): 245-7.

3. Underreker DF, Shepard RB, Schmidt CL, Crespi AM, Skarstad PM. Power sources for implantable pacemakers. In: Ellenbogen KA, Kay GN, Wilkoff BL. Clinical Cardiac Pacing. Philadelphia: WB Saunders, 1995; Cap. 4: 91-111.

4. Stokes KB, Kay GN. Artificial electric cardiac pacing. In: Ellenbogen KA, Kay GN, Wilkoff BL. Clinical Cardiac Pacing. Philadelphia: WB Saunders, 1995; Cap. 1: 1-37.

5. Gregoratos G, Cheitlin MD, Conill A, et al. ACC/AHA guidelines for implantation of cardiac pacemakers and antiarrhythmia devices: a report of the American College of Cardiology/American Heart Association Task Force on Practice Guidelines (Committee on Pacemaker Implantation). J Am Coll Cardiol 1998; 31 : 1175-209.

6. Berstein AD, Irwin ME, Parsonnet V, et al. Policy-conference report. antibradycardia-pacemaker follow-up: Effectiveness, needs and resources. Pacing Clin Electrophysiol 1994; 17:1714-29.

7. Griffin JC, SchuenemeyerTD, Hess KR, et al. Pacemaker follow-up: Its role in the detection and correction of pacemaker system malfuntion. Pacing Clin Electrophysiol 1986; 9: 387-91.

8. Irnich W, KramerE, Muller R. The progamming of cardiac pacemakers - wish and reality. Dtsch Med Wochenschr 1991; 116: 601-5.

9. Barold SS, Stokes K, Byrd CL, Venes R. Energy parametes in cardiac pacieng should be abandoned. Pacing Clin Electrophysiol 1997; 20: 111-21.
10. Tyers GOT, Brownlee RR. Power pulse generators, electrodes, and longevity. Prog Cardiovasc Dis 1981; 23: 421-34.

11. Crossley GH, Brinker JA, Reynolds D, et al. Steroid elution improves the stimulation threshold in an active-fixation atrial permanent pacing lead. A randomized, controlled study. Model 4068 Investigators. Circulation 1995; 92: 2935-9.

12. Schwaab B, Frohlig G, Schwerdt H. Telemetry guided pacemaker programming: impact of output amplitude and the use of low threshold leads on projected pacemaker longevity. Pacing Clin Electrophysiol 1998; 21(Pt 1): 2055-63.

13. Schwaab B, Schwerdt H, Heisel A, et al. Chronic ventricular pacing using an output amplitude of 1.0 Volts. Pacing Clin Electrophysiol 1997; 20: 2171-78.

14. Stamato NJ, O'Toole MF, Fetter JG, Enger EL. The safety and efficacy if chronic ventricular ventricular pacing at 1.6 volts using steroid eluting lead. Pacing Clin Electrophysiol 1992; 15: 248-51.

15. Klein HH, Knake W. Energy-conserving of VVI pacemakers: a telemetry-supported, long-term, follow-up study. Clin Cardiol 1990; 13: 409-13.

16. Gillis AM, McQuarrie DS, Wilson SL. The impact of pulse generator longevity on the long-term costs of cardiac pacing. Pacing Clin Electrophysiol 1990; 19: 1459-68.

17. Irnich W. Pacemaker-related patient mortality. Pacing Clin Electrophysiol 1999; 22: 1279-83.

18. Clarke M, Liu B, Schuller H, et al. Automatic adjustment of pacemaker stimulation output correlated with continuously monitored capture thresholds: a multicenter study. European Microny Study Group. Pacing Clin Electrophysiol 1998; 21: 1567-75. 